\title{
On Mesoscopic Forces and Quantized Conductance in Model Metallic Nanowires
}

\author{
Constantine Yannouleas and Uzi Landman* \\ School of Physics, Georgia Institute of Technology, Atlanta, Georgia 30332-0430 \\ Received: June 9, $1997^{\otimes}$
}

\begin{abstract}
Energetics and conductance in jellium-modeled nanowires are investigated using the local-density-functionalbased shell correction method. In analogy with studies of other finite-size fermion systems, e.g., simple metal clusters or ${ }^{3} \mathrm{He}$ clusters, we find that the energetics of the wire as a function of its radius (transverse reduced dimension) leads to formation of self-selecting magic wire configurations (MWCs, i.e., discrete sequence of wire radii with enhanced stability), originating from quantization of the electronic spectrum, namely formation of subbands that are the analogs of electronic shells in clusters. These variations in the energy result in oscillations in the force required to affect a transition from one MWC of the nanowire to another and are correlated directly with stepwise variations of the quantized conductance of the nanowire in units of $2 e^{2} / h$.
\end{abstract}

\section{Introduction}

Identification and understanding of the physical origins and systematics underlying the variations of material properties with size, form of aggregation, and dimensionality are some of the main challenges in modern materials research, of ever increasing importance in the face of the accelerated trend toward miniaturization of electronic and mechanical devices. While for over two decades studies of size-evolutionary patterns of materials have focused on atomic and molecular clusters ${ }^{1-3}$ in beams or embedded in inert matrices, more recent efforts concentrated on preparation, characterization, and understanding of finite solid-state structures. These include nanometer-scale metal and semiconductor nanocrystals, ${ }^{4-6}$ surface-supported structures and quantum dots, ${ }^{7}$ and nanoscale junctions or wires. ${ }^{8-27}$

Interestingly, it has emerged that concepts and methodologies developed in the context of isolated gas-phase clusters and atomic nuclei are often most useful for investigations of finitesize solid-state structures. In particular, it has been shown most recently ${ }^{25,27}$ through first principles molecular dynamics simulations that as metallic (sodium) nanowires are stretched to just a few atoms in diameter, the reduced dimensions, increased surface-to-volume ratio, and impoverished atomic environment lead to formation of structures made of the metal atoms in the neck, which can be described in terms of those observed in small gas-phase sodium clusters; hence, they were termed supported cluster-derived structures (cds). The above prediction of the occurrence of "magic-number" cds in nanowires, due to characteristics of electronic cohesion and atomic bonding in such structures of reduced dimensions, are directly correlated with the energetics of metal clusters, where magic-number sequences of clusters sizes, shapes, and structural motifs due to electronic and/or geometric shell effects have been long predicted and observed. . $^{2,3,28}$

Furthermore, these results lead one directly to conclude that other properties of nanowires, derived from their energetics, may be described using methodologies developed previously in the context of clusters. Indeed, in this paper, we show that certain aspects of the mechanical response (i.e., elongation force) and electronic transport (e.g., quantized conductance) in metallic nanowires can be analyzed using the local-density-approximation (LDA)-based shell correction method (SCM), developed and applied previously in studies of metal clusters. ${ }^{3,29}$ Specif-

\footnotetext{
${ }^{\otimes}$ Abstract published in Advance ACS Abstracts, July 15, 1997.
}

ically, we show that in a jellium-modeled, volume-conserving nanowire, variations of the total energy (particularly terms associated with electronic subband corrections) upon elongation of the wire lead to self-selection of a sequence of stable "magic" wire configurations (MWCs, specified in our model by a sequence of the wire's radii), with the force required to elongate the wire from one configuration to the next exhibiting an oscillatory behavior. Moreover, we show that due to the quantized nature of electronic states in such wires, the electronic conductance varies in a quantized stepwise manner (in units of the conductance quantum $g_{0}=2 e^{2} / h$ ), correlated with the transitions between MWCs and the above-mentioned force oscillations.

Prior to introducing the model studied in this paper, it is appropriate to briefly review certain previous theoretical and experimental investigations, which form the background and motivation for this study. Atomistic descriptions, based on realistic interatomic interactions and/or first-principles modeling and simulations, played an essential role in discovering the formation of nanowires and in predicting and elucidating the microscopic mechanisms underlying their mechanical, spectral, electronic, and transport properties.

Formation and mechanical properties of interfacial junctions (in the form of crystalline nanowires) have been predicted through early molecular-dynamics simulations, ${ }^{8}$ where the materials (gold) were modeled using semiempirical embeddedatom potentials. In these studies it has been shown that separation of the contact between materials leads to generation of a connective junction that elongates and narrows through a sequence of structural instabilities; at the early stages, elongation of the junction involves multiple slip events, while at the later stages, when the lateral dimension of the wire necks down to a diameter of about $15 \AA$, further elongation involves a succession of stress accumulation and fast relief stages associated with a sequence of order-disorder structural transformations localized to the neck region. $8,22,23$ These structural evolution patterns have been shown through the simulations to be protrayed in oscillations of the force required to elongate the wire, with a period approximately equal to the interlayer spacing. In addition, the "saw-toothed" character of the predicted force oscillations (see Figure $3 b$ in ref 8 and Figure 3 in ref 22) reflects the stress accumulation and relief stages of the elongation mechanism. Moreover, the critical resolved yield stress of gold nanowires has been predicted ${ }^{8,22}$ to be $\sim 4 \mathrm{GPa}$, which is over an order of 
magnitude larger than that of the bulk, and is comparable to the theoretical value for $\mathrm{Au}(1.5 \mathrm{GPa})$ in the absence of dislocations.

These predictions, as well as anticipated electronic conductance properties, ${ }^{8,9}$ have been corroborated in a number of experiments using scanning tunneling and force microscopy, ${ }^{8,10-12,14,19,24}$ break junctions, ${ }^{13}$ and pin-plate techniques ${ }^{15,22}$ at ambient environments, as well as under ultrahigh vacuum and/or cryogenic conditions. Particularly pertinent to our current study are experimental observations of the oscillatory behavior of the elongation forces and the correlations between the changes in the conductance and the force oscillations; see especially the simultaneous measurements of force and conductance in gold nanowires in ref 19 , where in addition the predicted "ideal" value of the critical yield stress has also been measured (see also ref 24).

The jellium-based model introduced in this paper, which by construction is devoid of atomic crystallographic structure, does not address issues pertaining to nanowire formation methods, atomistic configurations, and mechanical response modes [e.g., plastic deformation mechanisms, interplanar slip, ordering and disordering mechanisms (see detailed descriptions in refs 8, 22, and 23 and a discussion of conductance dips in ref 12), defects, mechanical reversibility, ${ }^{19,22}$ and roughening of the wire's morphology during elongation ${ }^{23}$ ], nor does it consider the effects of the above on the electron spectrum, transport properties, and dynamics. ${ }^{27}$ Nevertheless, as shown below, the model offers a useful framework for linking investigations of solid-state structures of reduced dimensions (e.g., nanowires) with methodologies developed in cluster physics, as well as highlighting certain nanowire phenomena of mesoscopic origins and their analogies to clusters.

\section{The Jellium Model for Metallic Nanowires: Theoretical Method and Results}

Consider a cylindrical jellium wire of length $L$, having a positive background with a circular cross section of radius $R$ $\ll L$. For simplicity, we restrict ourselves here to this symmetry of the wire cross section. Variations in the shape of the nanowire cross section serve to affect the degeneracies of the electronic spectrum ${ }^{20,26}$ without affecting our general conclusions. We also do not include here variations of the wire's shape along its axis. Adiabatic variation of the wire's axial shape introduces a certain amount of smearing of the conductance steps through tunneling, depending on the axial radius of curvature of the wire..$^{20,21,26}$ Both the cross-sectional and axial shape of the wire can be included in our model in a rather straightforward manner.

The principal idea of the SCM is the separation of the total LDA energy $E_{\mathrm{T}}(R)$ as $3,29,30$

$$
E_{\mathrm{T}}(R)=\tilde{E}(R)+\Delta E_{\mathrm{sh}}(R)
$$

where $\tilde{E}(R)$ varies smoothly as a function of the system size and $\Delta E_{\mathrm{sh}}(R)$ is an oscillatory term arising from the discrete quantized nature of the electronic levels. $\Delta E_{\mathrm{sh}}(R)$ is usually called a shell correction in the nuclear ${ }^{30}$ and cluster ${ }^{3,29}$ literature; we continue to use here the same terminology with the understanding that the electronic levels in the nanowire form subbands, which are the analog of electronic shells in clusters, where furthermore the size of the system is usually given by specifying the number of atoms $N$. The SCM method, which has been shown to yield results in excellent agreement with experiments $^{3,31,32}$ and self-consistent LDA calculations ${ }^{3,29}$ for a number of cluster systems, is equivalent to a Harris functional ( $\left.E_{\text {harris }}\right)$ approximation to the Kohn-Sham LDA with the input

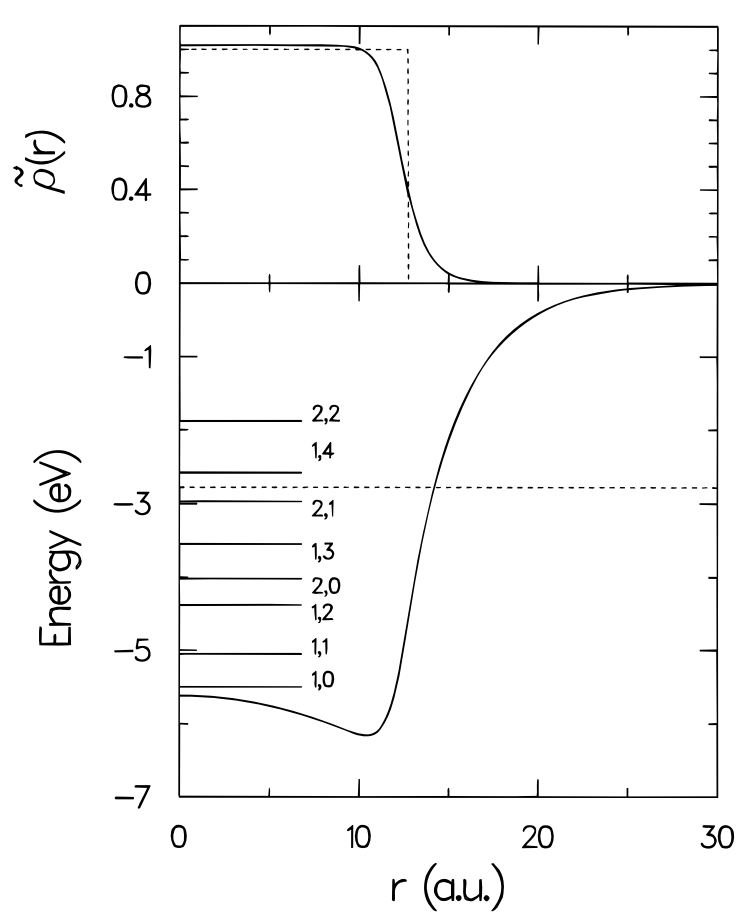

Figure 1. (Lower) $V_{\mathrm{ETF}}(r)$ potential for a sodium wire with a uniform jellium background of radius $R=12.7$ au, plotted versus the transverse radial distance from the center of the wire, along with the locations of the bottoms of the subbands (namely the transverse eigenvalues $\tilde{\epsilon}_{n m} ; n$ is the number of nodes in the radial direction plus one, and $m$ is the azimuthal quantum number of the angular momentum). The Fermi level is denoted by a dashed line. (Top) Jellium background volume density (dashed line) and the electronic volume density $\tilde{\rho}(r)$ (solid line, exhibiting a characteristic spillout) normalized to bulk values.

density obtained through variational minimization of an extended Thomas-Fermi (ETF) energy functional, $E_{\mathrm{ETF}}[\tilde{\rho}]$ (with the kinetic energy, $T_{\mathrm{ETF}}[\tilde{\rho}]$, given to fourth order gradients and the potential, $V_{\mathrm{ETF}}$, including the Hartree repulsion and exchangecorrelation and positive-background attractions as in LDA). The smooth contribution in eq 1 is identified with $E_{\mathrm{ETF}}[\tilde{\rho}]$. The optimized density ${ }^{33} \tilde{\rho}$ at a given radius $R$ is obtained under the normalization condition (charge neutrality) $2 \pi \int \tilde{\rho}(r) r \mathrm{~d} r=$ $\rho_{\mathrm{L}}^{(+)}(R)$, where $\rho_{\mathrm{L}}^{(+)}(R)=3 R^{2} /\left(4 r_{\mathrm{s}}^{3}\right)$ is the linear positive background density. Using the optimized $\tilde{\rho}$, one solves for the eigenvalues $\tilde{\epsilon}_{i}$ of the Hamiltonian $H=-\left(\hbar^{2} / 2 m\right) \nabla^{2}+V_{\mathrm{ETF}}[\tilde{\rho}]$, and the shell correction is given by

$$
\begin{aligned}
\Delta E_{\mathrm{sh}} & \equiv E_{\mathrm{harris}}[\tilde{\rho}]-E_{\mathrm{ETF}}[\tilde{\rho}] \\
& =\sum_{i=1}^{\text {occ }} \tilde{\epsilon}_{i}-\int \tilde{\rho}(\mathbf{r}) V_{\mathrm{ETF}}[\tilde{\rho}(\mathbf{r})] \mathrm{d} \mathbf{r}-T_{\mathrm{ETF}}[\tilde{\rho}]
\end{aligned}
$$

where the summation extends over occupied levels. Here the dependence of all quantities on the pertinent size variable (i.e., the radius of the wire $R$ ) is not shown explicitly. Additionally, the index $i$ can be both discrete and continuous, and in the latter case the summation is replaced by an integral.

Following the above procedure with a uniform background density of sodium $\left(r_{\mathrm{s}}=4 \mathrm{au}\right)$, a typical potential $V_{\mathrm{ETF}}(r)$ for $R$ $=12.7 \mathrm{au}$, where $r$ is the radial coordinate in the transverse plane, is shown in Figure 1, along with the transverse eigenvalues $\tilde{\epsilon}_{n m}$ and the Fermi level; to simplify the calculations of the electronic spectrum, we have assumed (as noted above) $R$ $\ll L$, which allows us to express the subband electronic spectrum as

$$
\tilde{\epsilon}_{n m}\left(k_{z} ; R\right)=\tilde{\epsilon}_{n m}(R)+\frac{\hbar^{2} k_{z}^{2}}{2 m}
$$




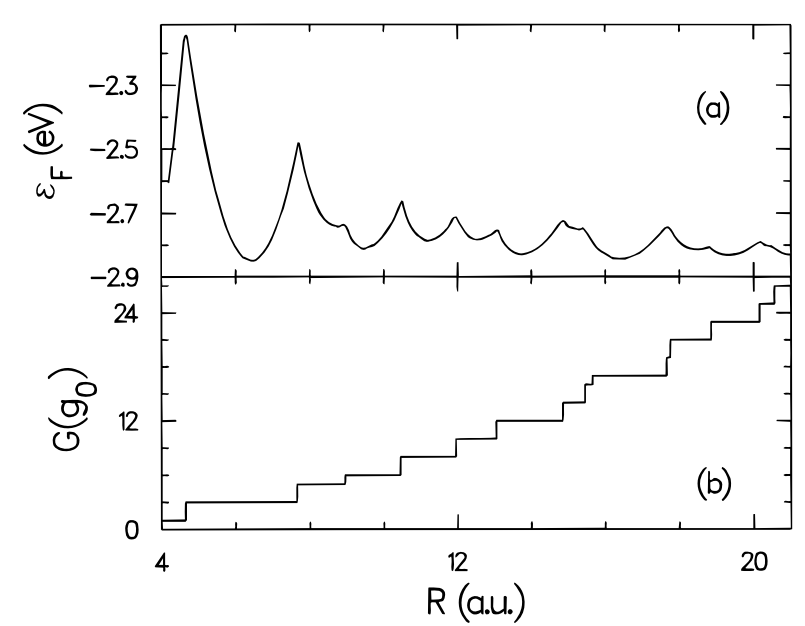

Figure 2. Variation of the Fermi energy $\epsilon_{\mathrm{F}}$ [shown in (a)] and of the conductance $G$ [shown in (b) in units of $g_{0}=2 e^{2} / h$ ], plotted versus the radius $R$, for a sodium nanowire. Note the coincidence of the cusps in $\epsilon_{\mathrm{F}}$ with the step rises of the conductance. The heights of the steps in $G$ reflect the subband degeneracies due to the circular shape of the wire's cross section.

where $k_{z}$ is the electron wavenumber along the axis of the wire (z).

As indicated earlier, taking the wire to be charge neutral, the electronic linear density, $\rho_{\mathrm{L}}^{(-)}(R)$, must equal the linear positive background density, $\rho_{\mathrm{L}}^{(+)}(R)$. The chemical potential (at $T=$ 0 the Fermi energy $\epsilon_{\mathrm{F}}$ ) for a wire of radius $R$ is determined by setting the expression for the electronic linear density derived from the subband spectra equal to $\rho_{\mathrm{L}}^{(+)}(R)$, i.e.

$$
\frac{2}{\pi} \sum_{n, m}^{\text {occ }} \sqrt{\frac{2 m}{\hbar^{2}}\left[\epsilon_{\mathrm{F}}(R)-\tilde{\epsilon}_{n m}(R)\right]}=\rho_{\mathrm{L}}^{(+)}(R)
$$

where the factor of 2 on the left is due to the spin degeneracy. The summand defines the Fermi wave vector for each subband, $k_{\mathrm{F}, n m}$. The resulting variation of $\epsilon_{\mathrm{F}}(R)$ versus $R$ is displayed in Figure $2 \mathrm{a}$, showing cusps for values of the radius where a new subband drops below the Fermi level as $R$ increases (or conversely as a subband moves above the Fermi level as $R$ decreases upon elongation of the wire). Using the Landauer expression for the conductance $G$ in the limit of no mode mixing and assuming unit transmission coefficients, $G(R)=g_{0} \sum_{n, m}$ $\Theta\left[\epsilon_{\mathrm{F}}(R)-\tilde{\epsilon}_{n m}(R)\right]$, where $\Theta$ is the Heaviside step function. The conductance of the nanowire, shown in Figure 2b, exhibits quantized stepwise behavior, with the step rises coinciding with the locations of the cusps in $\epsilon_{\mathrm{F}}(R)$, and the height sequence of the steps is $1 g_{0}, 2 g_{0}, 2 g_{0}, 1 g_{0}, \ldots$, reflecting the circular symmetry of the cylindrical wires' cross sections, ${ }^{9}$ as observed for sodium nanowires. ${ }^{13}$ Solving for $\epsilon_{\mathrm{F}}(R)$ (see eq 4), the expression for the sum on the right-hand-side of eq 2 can be written as

$$
\begin{aligned}
\sum_{i}^{\mathrm{occ}} \tilde{\epsilon}_{i} & =\frac{2}{\pi} \sum_{n, m}^{\mathrm{occ}} \int_{0}^{k_{\mathrm{F}, n m}} \mathrm{~d} k_{z} \tilde{\epsilon}_{\mathrm{nm}}\left(k_{z} ; R\right)= \\
& \frac{2}{3 \pi} \sum_{n, m}^{\mathrm{occ}}\left[\epsilon_{\mathrm{F}}(R)+2 \tilde{\epsilon}_{n m}(R)\right] \sqrt{\frac{2 m}{\hbar^{2}}\left[\epsilon_{\mathrm{F}}(R)-\tilde{\epsilon}_{n m}(R)\right]}
\end{aligned}
$$

which allows one to evaluate $\Delta E_{\text {sh }}$ (eq 2) for each wire radius $R$. Since the expression in eq 5 gives the energy per unit length, we also calculate $E_{\mathrm{ETF}}, T_{\mathrm{ETF}}$, and the volume integral in the second line of eq 2 for cylindrical volumes of unit height. To convert to energies per unit volume [denoted as $\epsilon_{\mathrm{T}}(R), \tilde{\epsilon}(R)$, and $\Delta \epsilon_{\mathrm{sh}}(R)$, all energies are further divided by the wire's cross-

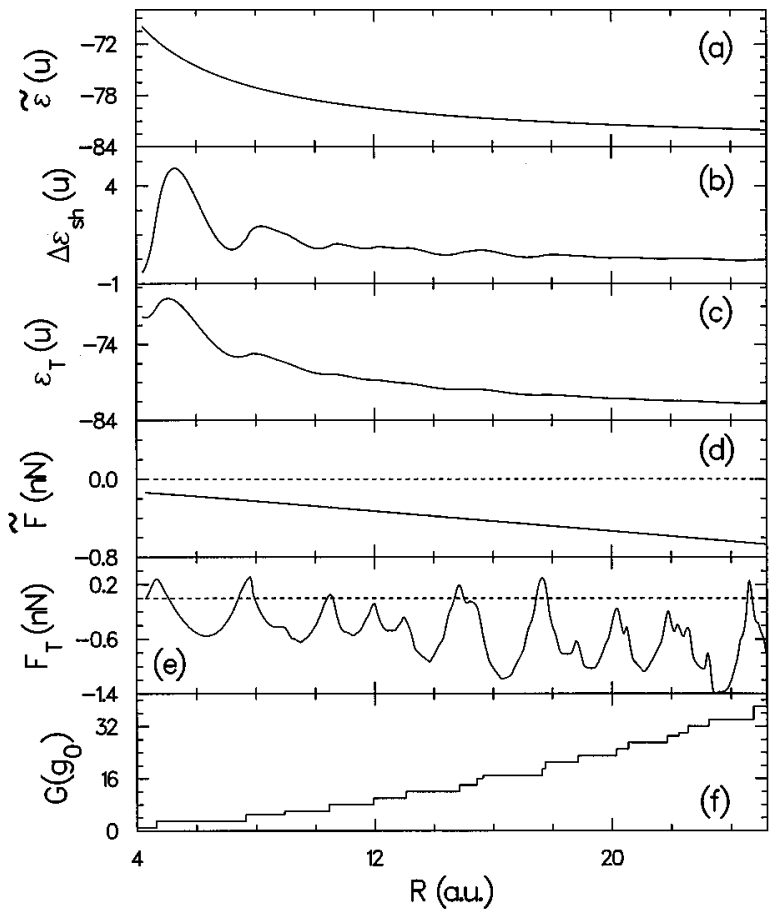

Figure 3. $(a-c)$ Smooth (a) and shell-correction (b) contributions to the total energy (c) per unit volume of the jellium-modeled sodium nanowire (in units of $u \equiv 10^{-4} \mathrm{eV} / \mathrm{au}^{3}$ ), plotted versus the radius of the wire (in au). Note the smaller magnitude of the shell corrections relative to the smooth contribution. (d, e) Smooth contribution (d) to the total force and the total force (e), plotted in units of $\mathrm{nN}$ versus the wire's radius. In (e), the zeroes of the force to the left of the force maxima occur at radii corresponding to the local minima of the energy of the wire (c). In (f), we reproduce the conductance of the wire (in units of $g_{0}=2 e^{2} / h$ ), plotted versus $R$. Interestingly, calculations of the conductance for the MWCs (i.e., the wire radii corresponding to the locations of the step rises) through the Sharvin-Weyl formula, ${ }^{18,26}$ corrected for the finite height of the confining potential ${ }^{18}$ (see lower panel of Figure 1), namely $G=g_{0}\left(\pi S / \lambda_{\mathrm{F}}^{2}-\alpha P / \lambda_{\mathrm{F}}\right)$, where $S$ and $P$ are the area and perimeter of the wire's cross section and $\lambda_{\mathrm{F}}$ is the Fermi wavelength $\left(\lambda_{\mathrm{F}}=12.91\right.$ au for Na) with $\alpha=0.1$ (see ref 18), yield results that approximate well the conductance values (i.e., the values at the bottom of the step rises) shown in (f).

sectional area, $\pi R^{2}$. The smooth contribution and the shell correction to the wire's energy are shown respectively in parts $a$ and $b$ of Figure 3 . The smooth contribution decreases slowly toward the bulk value $\left(-2.25 \mathrm{eV}\right.$ per atom $\left.{ }^{29}\right)$. On the other hand, the shell corrections are much smaller in magnitude and exhibit an oscillatory behavior. This oscillatory behavior remains visible in the total energy (Figure 3c) with the local energy minima occurring for values $R_{\min }$ corresponding to conductance plateaus. The sequence of $R_{\min }$ values defines the MWCs, that is, a sequence of wire configurations of enhanced stability.

From the expressions for the total energy of the wire [i.e., $\Omega \epsilon_{\mathrm{T}}(R)$, where $\Omega=\pi R^{2} L$ is the volume of the wire] and the smooth and shell (subband) contributions to it, we can calculate the "elongation force" (EF)

$$
\begin{gathered}
F_{\mathrm{T}}(R)=-\frac{\mathrm{d}\left[\Omega \epsilon_{\mathrm{T}}(R)\right]}{\mathrm{d} L}=-\Omega\left\{\frac{\mathrm{d} \tilde{\epsilon}(R)}{\mathrm{d} L}+\frac{\mathrm{d}\left[\Delta \epsilon_{\mathrm{sh}}(R)\right]}{\mathrm{d} L}\right\} \\
\equiv \tilde{F}(R)+\Delta F_{\mathrm{sh}}(R)
\end{gathered}
$$

Using the volume conservation, i.e., $\mathrm{d}\left(\pi R^{2} L\right)=0$, these forces can be written as $F_{\mathrm{T}}(R)=\left(\pi R^{3} / 2\right) \mathrm{d} \epsilon_{\mathrm{T}}(R) / \mathrm{d} R, \tilde{F}(R)=$ $\left(\pi R^{3} / 2\right) \mathrm{d} \tilde{\epsilon}(R) / \mathrm{d} R$, and $\Delta F_{\mathrm{sh}}(R)=\left(\pi R^{3} / 2\right) \mathrm{d}\left[\Delta \epsilon_{\mathrm{sh}}(R)\right] / \mathrm{d} R . \quad \tilde{F}(R)$ and $F_{\mathrm{T}}(R)$ are shown in Figure $3 \mathrm{~d}$,e. The oscillations in the force resulting from the shell-correction contributions dominate. 
In all cases, the radii corresponding to zeroes of the force situated on the left of the force maxima coincide with the minima in the potential energy curve of the wire, corresponding to the MWCs. Consequently, these forces may be interpreted as guiding the self-evolution of the wire toward the MWCs. Also, all the local maxima in the force occur at the locations of step rises in the conductance (reproduced in Figure 3f), signifying the sequential decrease in the number of subbands below the Fermi level (conducting channels) as the wire narrows (i.e., as it is being elongated). Finally the magnitude of the total forces is comparable to the measured ones (i.e., in the nanonewton range).

\section{Conclusions and Discussion}

We investigated energetics, conductance, and mesoscopic forces in a jellium modeled nanowire (sodium) using the localdensity-functional-based shell correction method. The results shown above, particularly the oscillations in the total energy of the wire as a function of its radius (and consequently the oscillations in the EF), the corresponding discrete sequence of magic wire configurations, and the direct correlation between these oscillations and the stepwise quantized conductance of the nanowires, originate from quantization of the electronic states (i.e., formation of subbands) due to the reduced lateral (transverse) dimension of the nanowires. In fact such oscillatory behavior and the appearance of "magic numbers" and "magic configurations" of enhanced stability are general characteristics of finite-size fermionic systems and are in direct analogy with those found in simple metal clusters (as well as in ${ }^{3} \mathrm{He}$ clusters ${ }^{32}$ and atomic nuclei ${ }^{30}$ ), where electronic shell effects on the energetics ${ }^{2,3,29,31}$ (and most recently shape dynamics ${ }^{34}$ of jelliummodeled clusters driven by forces obtained from shell-corrected energetics) have been studied for over a decade.

While these calculations provide a useful and instructive framework, we remark that they are not a substitute for theories where the atomistic nature and specific atomic arrangements are included $8,22,23,25,27$ in evaluation of the energetics (and dynamics) of these systems (see in particular refs 25 and 27 , where first-principles molecular-dynamics simulations of electronic spectra, geometrical structure, atomic dynamics, electronic transport, and fluctuations in sodium nanowires have been discussed).

Indeed, the atomistic structural characteristics of nanowires (including the occurrence of cluster-derived structures of particular geometries ${ }^{25,27}$ ), which may be observed through the use of high-resolution microscopy, ${ }^{35}$ influence the electronic spectrum and transport characteristics, as well as the energetics of nanowires and their mechanical properties and response mechanisms. In particular, the mechanical response of materials involves structural changes through displacement and discrete rearrangement of the atoms. The mechanisms, pathways, and rates of such structural transformations are dependent on the arrangements and coordinations of atoms, the magnitude of structural transformation barriers, and the local shape of the wire, as well as possible dependency on the history of the material and the conditions of the experiment (i.e., fast versus slow extensions). Further evidence for the discrete atomistic nature of the structural transformations is provided by the shape of the force variations (compare the calculated Figure $3 \mathrm{~b}$ in ref 8 and Figure 3 in ref 22 with the measurements shown in Figures 1 and 2 in ref 19) and the interlayer spacing period of the force oscillations when the wire narrows. While such issues are not addressed by our model, the mesoscopic (in a sense universal) phenomena described by it are of interest and may guide further research in the area of finite-size systems in the nanoscale regime.
Acknowledgment. This research was supported by a grant from the U.S. Department of Energy (Grant No. FG0586ER45234) and the AFOSR. Useful conversations with W. D. Luedtke, E. N. Bogachek, and R. N. Barnett are greatfully acknowledged. Calculations were performed at the Georgia Institute of Technology Center for Computational Materials Science.

\section{References and Notes}

(1) Clusters of Atoms and Molecules; Haberland, H., Ed.; Springer Series in Chemical Physics 52 and 57; Springer: Berlin, 1994.

(2) De Heer, W. A. Rev. Mod. Phys. 1993, 65, 611.

(3) Yannouleas, C.; Landman, U. In Large Clusters of Atoms and Molecules; Martin, T. P., Ed.; Kluwer: Dordrecht, 1996; p 131.

(4) Whetten, R. L.; Khoury, J. T.; Alvarez, M.; Murthy, S.; Vezmar, I.; Wang, Z. L.; Stephens, P. W.; Cleveland, C. L.; Luedtke, W. D.; Landman, U. Adv. Mater. 1996, 8, 428.

(5) Luedtke, W. D.; Landman, U. J. Phys. Chem. 1996, 100, 13323.

(6) Alivisatos, A. P. Science 1996, 271, 933.

(7) Atomic and Nanoneter-Scale Modification of Materials: Fundamentals and Applications; Avouris, P., Ed.; Kluwer: Dordrecht, 1993. Luedtke, W. D.; Landman, U. Phys. Rev. Lett. 1994, 73, 569.

(8) Landman, U.; Luedtke, W. D.; Burnham, N.; Colton, R. J. Science 1990, 248, 454.

(9) Bogachek, E. N.; Zagoskin, A. M.; Kulik, I. O. Fiz. Nizk. Temp. 1990, 16, 1404; Sov. J. Low Temp. Phys. (Engl. Transl.) 1990, 16, 796.

(10) Pascual, J. I.; Mendez, J.; Gomez-Herrero, J.; Baro, J. M.; Garcia, N.; Binh, V. T. Phys. Rev. Lett. 1993, 71, 1852.

(11) Olesen, L.; Laegsgaard, E.; Stensgaard, I.; Besenbacher, F.; Schiotz, J.; Stoltze, P.; Jacobsen, K. W.; Norskov, J. N. Phys. Rev. Lett. 1994, 72, 2251.

(12) Pascual, J. I.; Mendez, J.; Gomez-Herrero, J.; Baro, J. M.; Garcia, N.; Landman, U.; Luedtke, W. D.; Bogachek, E. N.; Cheng, H.-P. Science 1995, 267, 1793.

(13) Krans, J. M.; van Ruitenbeek, J. M.; Fisun, V. V.; Yanson, I. K.; de Jongh, L. J. Nature 1995, 375, 767.

(14) Smith, D. P. E. Science 1995, 269, 371.

(15) Costa-Kramer, J. L.; Garcia, N.; Garcia-Mochales, P.; Serena, P. A. Surf. Sci. 1995, 342, 11144.

(16) Bratkovsky, A. M.; Sutton, A. P.; Todorov, T. N. Phys. Rev. B 1995, 52, 5036 .

(17) Lang, N. D. Phys. Rev. B 1995, 52, 5335

(18) Garcia-Martin, A.; Torres, J. A.; Saenz, J. J. Phys. Rev. B 1996 54,13448 .

(19) Rubio, G.; Agrait, N.; Vieira, S. Phys. Rev. Lett. 1996, 76, 2302. (20) Scherbakov, A. G.; Bogachek, E. N.; Landman, U. Phys. Rev. B 1996, 53, 4054.

(21) Bogachek, E. N.; Scherbakov, A. G.; Landman, U. Phys. Rev. B 1996, 53, R13246.

(22) Landman, U.; Luedtke, W. D.; Salisbury, B. E.; Whetten, R. L. Phys. Rev. Lett. 1996, 77, 1362.

(23) Landman, U.; Luedtke, W. D.; Gao, J. Langmuir 1996, 12, 4514.

(24) Stalder, A.; Durig, U. Appl. Phys. Lett. 1996, 68, 637; Nature, in press.

(25) (a) Landman, U.; Barnett, R. N.; Luedtke, W. D. Z. Phys. D 1997, 40,282 . (b) The original predictions pertaining to formation of cds and analogies to cluster phenomena (e.g. magic numbers) have been presented by one of us (U.L.) at ISSPIC8 (Copenhagen, July 1996, see ref 25a), and at the NATO ARW on Nanowires (Madrid, September 1996); see Proceeding paper: Landman, U.; Luedtke, W. D.; Barnett, R. N. In Nanowires; Serena, P. A., Garcia, N., Eds.; Kluwer: Dordrecht, 1997. Subsequently and after completion of the work in this Letter, we learned about research related to some of these issues being pursued by van Ruitenbeek, J. M., et al.; Stafford, C. A., et al.; and Shekhter, R. I., et al.

(26) Bogachek, E. N.; Scherbakov, A. G.; Landman, U. Phys. Rev. B 1997, 56, 1065.

(27) Barnett, R. N.; Landman, U. Nature 1997, 387, 788.

(28) Martin, T. P. Phys. Rep. 1996, 273, 199.

(29) Yannouleas, C.; Landman, U. Phys. Rev. B 1993, 48, 8376; Chem. Phys. Lett. 1993, 210, 437.

(30) Bohr, A; Mottelson, B. R. Nuclear Structure; Benjamin: Reading, MA, 1975. Strutinsky, V. M. Nucl. Phys. A 1967, 95, 420; Nucl. Phys. A 1968, 122,1 .

(31) Yannouleas, C.; Landman, U. Phys. Rev. B 1995, 51, 1902; Phys. Rev. Lett. 1997, 78, 1424.

(32) Yannouleas, C.; Landman, U. J. Chem. Phys. 1996, 105, 8734

(33) For a description of the input trial density and the local exchangecorrelation functional, as well as the expression for the kinetic-energy functional, see refs 3 and 29.

(34) Yannouleas, C.; Landman, U. Submitted for publication.

(35) Kizuka, T.; Yamada, K.; Degachi, S.; Naruse, M.; Tanaka, N. Phys Rev. B 1997, 55, R7398. 